Case Report

\title{
Melioidosis presenting with periorbital cellulitis and eyelid abscess in Sarawak, Malaysian Borneo - A Case Report
}

\author{
Chee Yik Chang ${ }^{1}$ \\ Sri Lankan Journal of Infectious Diseases 2019 Vol.9 (2):160-164 \\ DOI: http://doi.org/10.4038/sljid.v9i2.8259
}

\begin{abstract}
Melioidosis is an infectious disease caused by the Gram-negative bacillus Burkholderia pseudomallei and presents with a broad spectrum of clinical manifestations and organ involvement. Ocular manifestation in melioidosis is rare. We present a case of periorbital cellulitis and eyelid abscess caused by $B$. pseudomallei in a diabetic patient successfully treated with antibiotic and surgical drainage.
\end{abstract}

Keywords: Melioidosis, Burkholderia pseudomallei, Periorbital cellulitis, Eyelid abscess

\section{Introduction}

Melioidosis is caused by Burkholderia pseudomallei, a Gram negative bacillus found in soil and water. It is an endemic disease in northern Australia and Southeast Asian countries, especially Thailand and Malaysia. Despite the availability of effective antimicrobial therapy in the treatment of melioidosis, the mortality rate remains high. ${ }^{1}$ Melioidosis is difficult to diagnose because of its diverse clinical presentation and can affect almost any organ. The manifestations of melioidosis are highly variable, ranging from pneumonia, septic arthritis, liver or splenic abscesses, pericardium and parapharyngeal space abscesses, skin abscesses, pan ophthalmitis to fatal septicaemia. ${ }^{2}$ Ocular involvement in melioidosis is rare and the prevalence was estimated at $0.49-1.02 \% .^{3}$ A 23 -year retrospective review of 16 cases of ocular involvement in melioidosis in Thailand revealed orbital cellulitis as the most common manifestation ( 7 cases) followed by endophthalmitis ( 4 cases), preseptal cellulitis (2 cases), pan ophthalmitis ( 2 cases), and pan uveitis ( 1 case). ${ }^{3}$ Melioidosis can also present with lid abscess in isolation without orbital cellulitis. ${ }^{4}$

\section{Case Presentation}

A 58-year-old male farmer presented in May 2018 to Kapit Hospital, Sarawak with fever and right eye swelling of 1 week duration. His past medical history included bacteraemic melioidosis which was diagnosed in 2016. At that time, he received

${ }^{1}$ Department of Medicine, Kapit Hospital, Ministry of Health, 96800 Kapit, Sarawak, Malaysia Address for correspondence: Dr Chee Yik Chang, Department of Medicine, Kapit Hospital, Ministry of Health, 96800 Kapit, Sarawak, Malaysia. Telephone: +60182856630 Email: ccyik28@gmail.com

https://orcid.org/0000-0002-3104-8168

Received 22 July 2019 and revised version accepted 10 September 2019 
intravenous ceftazidime as intensive phase therapy, followed by eradication therapy with oral trimethoprim-sulfamethoxazole. He was also diagnosed with type 2 diabetes mellitus during that hospitalization and started on oral anti-diabetic medications. However, he had defaulted on his medications for 1 year.

Upon arrival to the hospital, his general condition was stable and the vital signs were normal. The random blood sugar was $31.2 \mathrm{mmol} / \mathrm{L}$. There was periorbital swelling of the right eye with swelling and erythema of the upper and lower eyelids. The conjunctiva appeared normal. The visual acuity of the right eye was $6 / 15$ while vision of the left eye was reduced to finger counting. The remainder of systemic examination was unremarkable.

Haematological analysis showed a haemoglobin of $15.6 \mathrm{~g} / \mathrm{dL}$, white blood cell count of $7.4 \times 10^{3} / \mu \mathrm{L}$ and platelet count of $162 \times 10^{3} / \mu \mathrm{L}$. His renal and liver function tests were within normal limits. The chest radiograph and abdominal ultrasonography did not reveal any abnormality. Our initial working diagnosis was right periorbital cellulitis. He was started on intravenous ampicillin-sulbactam 1.5 gram 8-hourly. However, persistent fever was observed over the next 3 days. At this point, melioidosis was suspected in view of multiple risk factors including diabetes mellitus, occupational exposure and previous history of melioidosis. The antibiotic regime was escalated to intravenous ceftazidime 2 gram 8 -hourly to treat for melioidosis.

He was also referred to the ophthalmologist for an opinion. Computed tomography (CT) of the orbit showed right periorbital cellulitis (Fig. 1).

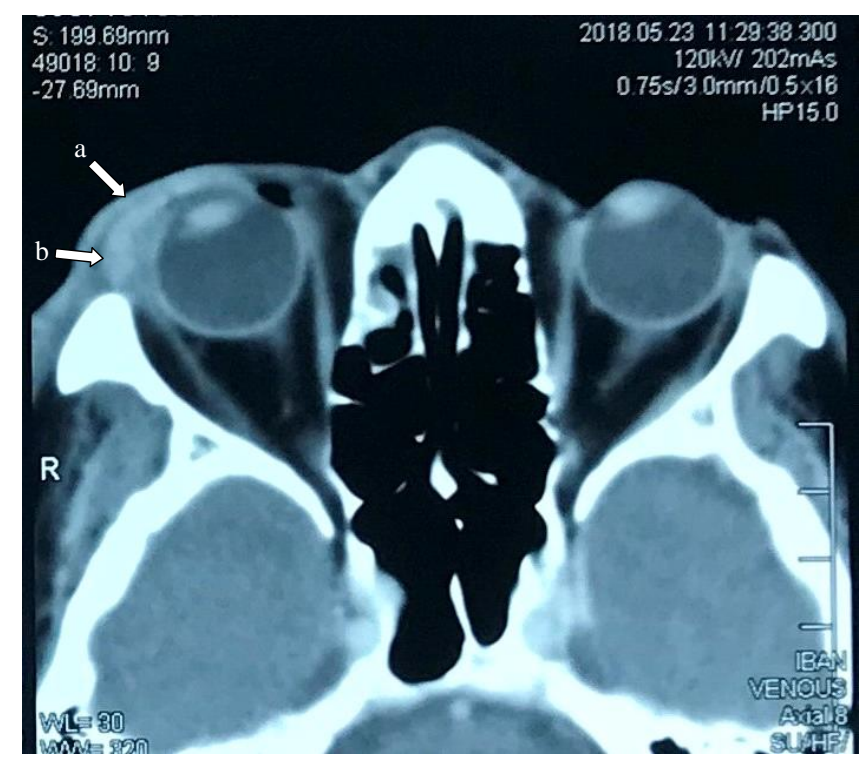

Fig. 1 Axial CT scan of orbit

(a) right periorbital swelling with soft tissue thickening

(b) increased attenuation of fat planes of right periorbital area while bilateral globes, extraocular muscles and optic nerve are preserved.
Detailed ophthalmic examination showed mild non-proliferative diabetic retinopathy in both eyes and increased cup-to-disc ratio of the left eye. As a result, floater only vitrectomy of the left eye was done by the ophthalmology team.

On day 8 of hospitalization, he developed an abscess over the right upper eyelid. Incision and drainage of the abscess was performed in which copious amounts of pus was drained and sent for microbiological analysis. The cultures of blood and pus from the eyelid abscess yielded $B$. pseudomallei, grown on modified Ashdown selective culture medium. The organism was susceptible to ceftazidime, amoxicillin-clavulanic acid and trimethoprim-sulfamethoxazole. The identification of $B$. pseudomallei was confirmed by a positive PCR assay specific for the detection of B. pseudomallei. 
He completed a 2-week course of intravenous ceftazidime. The fever completely settled on day 10 of hospitalization and the right eye swelling progressively reduced. His blood glucose control was good while on insulin therapy. He completed eradication therapy consisting of trimethoprim-sulfamethoxazole and doxycycline for 20 weeks. During a follow-up review at the medical clinic, he was well and showed no signs of disease recurrence. The right eye swelling had totally resolved, and visual acuity returned to baseline levels.

\section{Timeline of clinical progression}

\begin{tabular}{|c|c|c|}
\hline Time & Events & Actions/treatments \\
\hline Day 1 & $\begin{array}{l}\text { Admission for fever and right eye } \\
\text { swelling for } 1 \text { week; treated as right } \\
\text { periorbital cellulitis }\end{array}$ & $\begin{array}{l}\text { IV ampicillin-sulbactam } 1.5 \text { gram } \\
\text { 8-hourly }\end{array}$ \\
\hline Day 3 & $\begin{array}{l}\text { Persistent fever and suspected } \\
\text { melioidosis }\end{array}$ & IV ceftazidime 2 gram 8 -hourly \\
\hline Day 4 & $\begin{array}{l}\text { CT orbit showed right periorbital } \\
\text { cellulitis }\end{array}$ & $\begin{array}{l}\text { Co-managed by ophthalmology } \\
\text { team }\end{array}$ \\
\hline Day 5 & Blood culture grew B. pseudomallei & \\
\hline Day 8 & Appearance of right eyelid abscess & $\begin{array}{l}\text { Incision and drainage (pus culture } \\
\text { grew } B \text {. pseudomallei) }\end{array}$ \\
\hline Day 10 & $\begin{array}{l}\text { Resolution of fever and reducing right } \\
\text { eye swelling }\end{array}$ & Antibiotic continued \\
\hline Day 14 & Completed IV ceftazidime for 14 days & $\begin{array}{l}\text { Discharged home well with } \\
\text { trimethoprim-sulfamethoxazole } \\
\text { and doxycycline }\end{array}$ \\
\hline
\end{tabular}

\section{Discussion}

Melioidosis can present with a wide spectrum of clinical presentations. Pneumonia was the principal presentation of melioidosis in approximately half of the cases. Less common presentations include genitourinary infection, skin infection, bacteraemia without evident focus, septic arthritis or osteomyelitis and neurological melioidosis. ${ }^{5}$ Ocular manifestation in melioidosis is rare. There have been several case reports previously on corneal ulcers, orbital cellulitis and endophthalmitis caused by $B$. Pseudomallei. ${ }^{6-8}$ Melioidosis presenting with periorbital cellulitis and eyelid abscess has not been reported previously.

Periorbital cellulitis, also known as preseptal cellulitis is an infection of the eyelid and superficial periorbital soft tissues without the involvement of the globe and orbit. ${ }^{9}$ Patients with periorbital cellulitis generally do not require surgical intervention except in cases of eyelid abscess where drainage of abscess is recommended in addition to antibiotic treatment. ${ }^{9}$ Culture of the material is important to confirm the diagnosis and as a guide to appropriate antibiotic therapy. ${ }^{10}$

There are 3 modes of acquisition of $B$. pseudomallei which could result in melioidosis infection, namely inhalation, ingestion, and inoculation. ${ }^{2}$ The patient described in this case was a farmer who had constant exposure to soil or water in which this organism is found. We postulated that the most probable mode of acquisition was through direct inoculation into the affected eye causing primary ocular melioidosis and later, blood 
stream infection. This is consistent with the observation that most cases of ocular melioidosis are associated with B. pseudomallei bacteraemia, as reported by Yaisawang et al. ${ }^{3}$

Early diagnosis and prompt treatment are crucial for a favourable outcome. Periorbital cellulitis, if not treated promptly, can extend posteriorly into the orbit causing orbital cellulitis, subperiosteal abscess or orbital abscess. These would potentially lead to significant visual and central nervous system complications. ${ }^{9}$ In this case, our patient achieved resolution of periorbital cellulitis and eyelid abscess without any long term ophthalmic complication following early diagnosis of melioidosis, effective antibiotic therapy and surgical drainage.

\section{Conclusion}

Diagnosis of melioidosis should be considered in patients who present with periorbital cellulitis or eyelid abscess in areas where melioidosis is endemic. Surgical drainage of the abscess is an important part of management besides standard antibiotic treatment.

\section{Acknowledgements}

I would like to thank the Director General of Health Malaysia for his permission to publish this article. The author thanks the Director of Kapit Hospital, Dr Hii King Ching for her guidance and support, Dr Tan Li Mun, Ophthalmologist at Sibu Hospital for her expert opinion, and all staff of Kapit and Sibu Hospitals involved in the care of this patient.

Conflict of interest: The author declares that there are no conflicts of interest regarding the publication of this paper.

Consent for publication: Written informed consent for publication of the clinical details and clinical images was obtained from the patient.

\section{References}

1. Wiersinga WJ, van der Poll T, White NJ, et al. Melioidosis: insights into the pathogenicity of Burkholderia pseudomallei. Nat Rev Microbiol 2006; 4(4):272-82. doi:https://doi.org/038/nrmicro1385

2. Cheng AC, Currie BJ. Melioidosis: epidemiology, pathophysiology, and management. Clin Microbiol Rev 2005; 18(2):383-416. doi: https://doi.org/10.1128/CMR.18.2.383-416.2005

3. Yaisawang S, Asawaphureekorn S, Chetchotisakd P, et al. Ocular involvement in melioidosis: a 23-year retrospective review. J Ophthalmic Inflamm Infect 2018; 8(1):5. doi: http://doi.org/10.1186/s12348-018-0147-6

4. Wadwekar B, Suresh Ninan R, Bhat S, et al. Lid abscess: An unusual presentation of melioidosis. AMJ 2018; 11(6):322-5. doi:https://doi.org/10.21767/AMJ.2017.2809

5. Currie BJ, Ward L, Cheng AC. The epidemiology and clinical spectrum of melioidosis: 540 cases from the 20 year Darwin prospective study. PLoS Negl Trop Dis 2010; 4(11):e900. doi: https://doi.org/10.1371/journal.pntd.0000900

6. Siripanthong S, Teerapantuwat S, Prugsanusak W, et al. Corneal Ulcer caused by Pseudomonas pseudomallei: Report of Three Cases. Clin Infect Dis 1991; 13(2):335-7. doi: https://doi.org/10.1093/clinids/13.2.335 
7. Wong PK, Ng PH. Melioidosis presenting with orbital cellulitis. Singapore Med J 1996; 37(2):220-1. URL: https://www.ncbi.nlm.nih.gov/pubmed/8942270

8. Chen KJ, Sun MH, Hou CH, et al. Burkholderia pseudomallei Endophthalmitis. J Clin Microbiol 2007; 45(12):4073-4. doi: https://doi.org/10.1128/JCM.01467-07

9. Lee S, Yen MT. Management of preseptal and orbital cellulitis. Saudi J Ophthalmol 2011; 25(1):21-9. doi: https://doi.org/10.1016/J.SJOPT.2010.10.004

10. Inglis TJJ, Rolim DB, Rodriguez JLN. Clinical guideline for diagnosis and management of melioidosis. Rev Inst Med Trop Sao Paulo 2006; 48(1):1-4. doi: https://doi.org/ 10.1590/S0036-46652006000100001 